\title{
Molecular Docking Studies of Flavonoids of Noni Fruit (Morinda citrifolia L.) to Peroxisome Proliferator-Activated Receptor-Gamma (PPAR $\gamma$ )
}

\author{
Fikry Awaluddin ${ }^{1}$, Andrianopsyah Mas Jaya Putra ${ }^{2}$, Supandi $^{3 *}$ \\ ${ }^{I}$ School of Pharmacy, State Islamic University Syarif Hidayatullah, South Tangerang 15412, Indonesia. \\ ${ }^{2}$ Chemical Research Center - LIPI, PUSPITEK, South Tangerang 15314, Indonesia. \\ ${ }^{3}$ Faculty of Pharmacy, University of Muhammadiyah Prof. DR. Hamka, East Jakarta 13460, Indonesia.
}

Received: 23 September 2014 / Accepted: 30 November 2014

\begin{abstract}
:
Diabetes is a chronic disease that occurs either when the pancreas does not produce enough insulin or when the body cannot effectively use the produced insulin. Morinda citrifolia has been widely used as herbal medicine to prevent and to treat diabetes as well as health supplements. It contains flavonoids which may act as an antidiabetic agent. One of the receptors that play a role in the treatment of diabetes is peroxisome proliferator-activated receptor - gamma (PPAR $\gamma$ ) which responsible for insulin sensitization in adipose tissue. In this study, the interaction beetween flavonoids contained in noni fruit as ligands, and PPAR $\gamma$ was investigated by using the Autodock Vina software. Some flavonoids such as quercetin, kaempferol, nicotifloroside, narcissoside, and rutin were included in this study. Rosiglitazone, a known ligand for PPAR $\gamma$ was used as the standard. The result revealed that all the selected flavonoids showed binding energy ranging between $-8,1$ $\mathrm{kcal} / \mathrm{mol}$ to $-8,5 \mathrm{kcal} / \mathrm{mol}$. Eventhough, when compared with rosiglitazone $(-8.9 \mathrm{kcal} / \mathrm{mol})$, the value of the binding energy of flavonoids was higher, but close to rosiglitazone. This suggests that those flavonoid compounds may show comparable potential with rosiglitazone as antidiabetic agent.
\end{abstract}

Key words: Diabetes, morindacitrifolia, PPAR, flavonoid, molecular docking

\section{Introduction}

Diabetes is a chronic disease that occurs either when the pancreas does not produce enough insulin or when the body cannot effectively use the insulin it produces. Data from WHO in 2013 showed that 347 million people worldwide suffer from diabetes. Indonesia is the 7th country with the highest number of diabetics in the world. By 2030, the estimated population of diabetics in Indonesia reached 11.8 million, with the growth percentage of $6 \%$ per year which far exceeds the growth of overall Indonesia's population [1].

PPAR $\gamma$ plays a role in insulin sensitization process, and is used as a therapeutic target of type 2 diabetes mellitus. Thiazolidinediones is a class of antidiabetic agent works by binding to PPAR $\gamma$, thereby increasing insulin stimulation by glucose transporter receptor 4 (GLUT4) and glycogen synthesis which leads to increased insulin signaling and insulin sensitivity [2].

Morinda citrifolia, or noni fruit commonly called pace/noni, had been widely used by the public as prevention and treatment of diabetes as well as health supplements. Several previous studies stating that Morinda citrifolia contains approximately 200 chemical compounds which have been identified and isolated from various parts of this plant. One of them is a flavonoid compound found in the fruit, flowers, and leaves [3].

The ethanol extract of Morinda citrifolia can lower blood sugar levels due to antioxidant activity of flavonoid contained in the plant [4]. Quersetin can regenerate the pancreas and may increase the release of insulin in diabetic rats induced by streptozotosin [5]. In the meantime, the flavonoid compounds found in Citrus sp. [6] by the method of molecular docking, and resulted in a statement that the flavonoid compounds have good docking results on several protein targets related to diabetes, such as Peroxisome Proliferator-Activated Receptor-Gamma (PPAR $\gamma$ ) which responsible for insulin sensitization on the adipose tissue.

In this research, some flavonoids of Noni Fruit (Morinda citrifolia), including quercetin, kaempferol, nicotifloroside, narcissoside, and rutin as ligands for $\operatorname{PPAR} \gamma$ were investigated applying molecular docking as preliminary study in identifying potential candidate of antidiabetic drugs. Rosiglitazone was choosen as a positive control.

\footnotetext{
*Corresponding author: Supandi,

E-mail: supandi_19@yahoo.co.id
} 


\section{Experimental}

Preparation of three-dimensional structure of target protein

The three-dimensional structure of PPAR $\gamma$ (PDB ID 2PRG) was obtained from Protein Data Bank, (http://www.rcsb.org/pdb). Macromolecular proteins were separated from the solvent and ligand or nonstandard residues. The separation of macromolecules from the unneeded molecules were performed using the Discovery Studio 3.5 Visualizer. Protein receptor that had been separated from the residues was optimized with Autodock Tools (ADT) v1.5.6. The optimization include: the addition of hydrogen atoms to macromolecule and setting the grid box parameters. The size of grid box was set at $28 \times 28 \times 28(\mathrm{x}, \mathrm{y}, \mathrm{z})$ using $1.000 \AA$. The center of the grid box was put at $52.734 \times-3.774 \times 34.258(\mathrm{x}, \mathrm{y}, \mathrm{z})$. These results are saved in a format pdbqt.

\section{Preparation of ligands}

Rosiglitazone (CID 77999) used as a standard and flavonoid compounds from Morindacitrifolia, including quercetin (CID 5280343), kaempferol (CID 5280863), rutin (CID 5280805), nicotifloroside (CID 5318767), and narcissoside (CID 5481663) were downloaded from Pubchem (http://PubChem.ncbi.nlm.nih.gov) with .sdf format. Ligands' format were then converted into .pdb by using Open Babel. The structure of ligands that had been converted were further optimized with Autodock Tools by adding gasteiger charge. These results are saved in a format .pdbqt. MarvinSketch was used to perform QSAR studies in order to identify important molecular properties of ligands. Various properties of ligands such as $\log \mathrm{P}$, molecular weight, $\mathrm{H}$ bond donors, $\mathrm{H}$ bond acceptors were obtained. Lipinski's Rule of Five was then applied to select probable ligands.

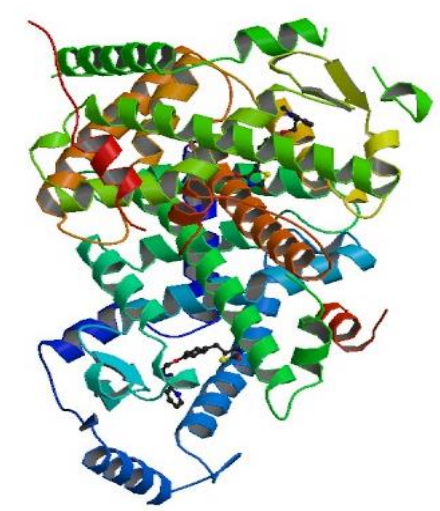

Figure 2. 3D Structure of PPAR $\gamma$.

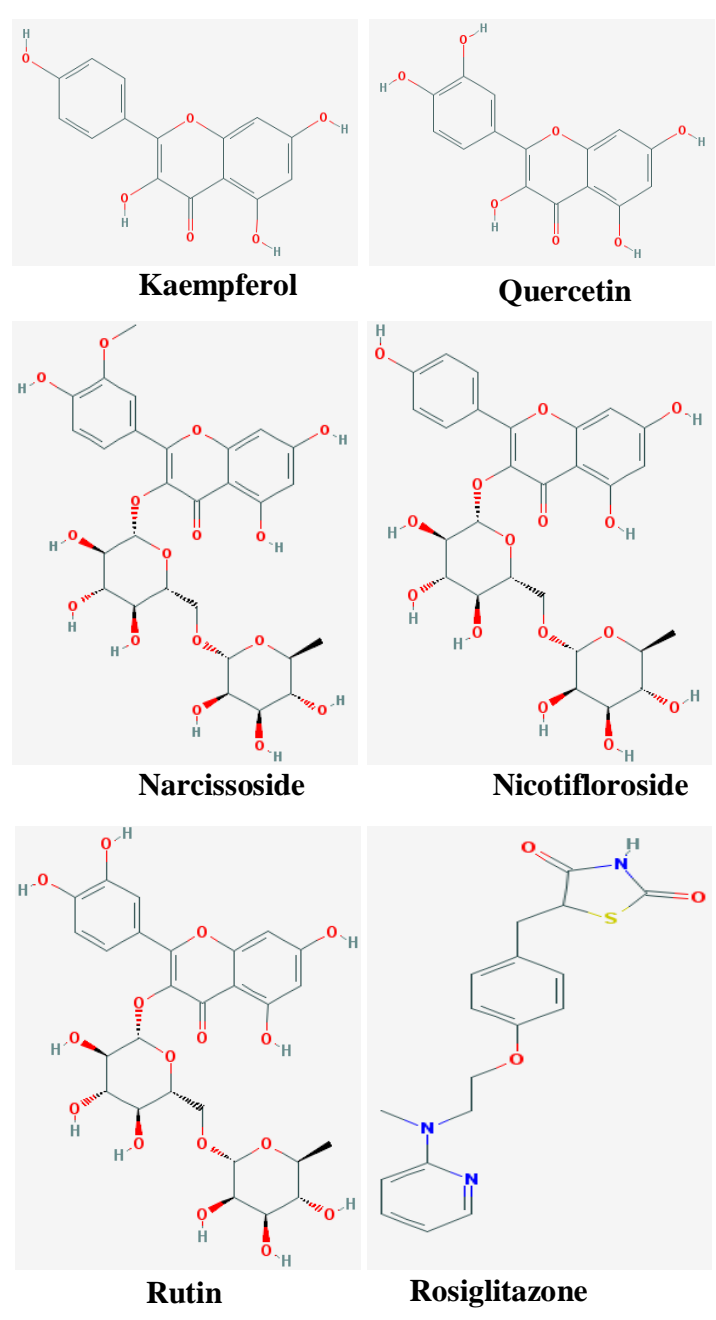

Figure 1. Structure of ligands.

\section{Molecular Docking Analysis}

The docking analysis of citrus flavonoids were carried out by Autodock Vina program. Vina ran through the Command prompt. Vina configuration settings file was center_x $=52.734$; center_y $=-3.774$; center_z $=34.258$ size_ $\mathrm{x}=28 ;$ size_y $=28 ;$ size_ $\mathrm{z}=28$, and exhaustiveness $=8$. The results of docking calculations seen in the output were in notepad format. Determination of the conformation of the ligand docking results were done by selecting the ligand conformation that showed the lowest binding energy (best pose). The position and orientation of ligands in protein receptor and the interaction with amino acids that bound to the ligand were analyzed and visualized with AutoDock tools.

\section{Results and Discussion}

Docking results were ranked by $\Delta G_{\text {bind }}$ best value (lowest energy). Low $\Delta G_{\text {bind }}$ energy indicates that the conformation formed was stable, whereas high $\Delta G_{\text {bind }}$ energy indicates that less stable complex was formed. 
As shown in Table 1, flavonoids showed binding energy in the range $-8.1 \mathrm{kcal} / \mathrm{mol}$ to $-8.5 \mathrm{kcal} / \mathrm{mol}$. Rutin produced the best value than other flavonoids. Even though, when compared with rosiglitazone, the value of the binding energy of flavonoids was still higher, but close to rosiglitazone $(-8.9 \mathrm{kcal} / \mathrm{mol})$. This proves that flavonoids may show comparable potential with rosiglitazone as antidiabetic agent.

Table 1 shows the protein residues that interact with the ligand. Hydrogen bond and hydrophobic contacts play an important role in the interaction between the ligands and the target protein. The best possible binding mode of all the ligands in target protein is displayed in Figure 3-8 by using AutoDock tools.

The design aims to find drug molecule ligands that can interact effectively to the target receptor. But it does not mean that the compound will be active when given orally. On its way to the target, the drug will experience some of the events are called pharmacokinetics, including absorption, distribution, metabolism, and excretion (ADME). Pharmacokinetics should be considered in the design of new drugs. There will be no drug target interaction and hence no biological activity triggered by the drug when the drug molecule never reach the target. As an approach, to design orally active drug, this drug must meet the 'Lipinski's Rule of Five' which states that [7]:

a) Its molecular weight should be less than 500 .

b) The compound's lipophilicity, expressed as $\log \mathrm{P}$ should be less than 5 .

c) The number of groups as hydrogen bond donor should be less than 5 .

d) The number of groups as hydrogen bond acceptor should be less than 10 .

Applying this rule, five flavonoid compounds and rosiglitazone that had been docked on the target protein were further investigated using Marvinsketch software to determine whether these compounds meet the Rule of Five of Lipinski. The results were displayed in Table 2. The table shows that from five flavonoid compounds, only quercetin and kaempferol which meet the four criteria of 'Lipinski's Rule, while the other three compounds violent the rule. This means that only quercetin and kaemperol that may be systemically absorbed and hence bioavailable when orally administered.

Table 1. $\Delta G_{\text {bind }}$ values and ligand interaction with residues

\begin{tabular}{lccl}
\hline Ligand & $\begin{array}{c}\Delta G_{\text {bind }} \\
(\mathrm{kcal} / \mathrm{mol})\end{array}$ & Hydrogen Bond & \multicolumn{1}{c}{ Hydrophobic Contact } \\
Rosiglitazone & -8.9 & Ser289 & $\begin{array}{l}\text { Leu228, Met329, Leu330, Leu333, Ile326, } \\
\text { Arg288, Ala292, Cys285, Gln286, His449, } \\
\text { Phe363 }\end{array}$ \\
\hline Rutin & -8.5 & Cys285, Ser289 & $\begin{array}{l}\text { Ala292, Glu291, Glu343, Ser342, Arg288, } \\
\text { Leu330, Leu33 }\end{array}$ \\
\hline Nicotifloroside & -8.4 & Glu291, Ser342 & $\begin{array}{l}\text { Ala292, Cys285, Arg288, Glu343, Ile341, } \\
\text { Ile326, Leu330, Leu333 }\end{array}$ \\
Narcissoside & -8.3 & Ser289, Glu343, Met348 & $\begin{array}{l}\text { Tyr327, Phe363, Met364, Val339, Ile281, } \\
\text { Leu330, Arg280,Ile341, Leu333, Ile326, } \\
\text { Cys285, Gly284, Ser342, Arg288 }\end{array}$ \\
\hline Quercetin & -8.1 & Ile326 & \begin{tabular}{l} 
Met329, Arg288, Ile341, Leu333 \\
\hline Kaempferol
\end{tabular} \\
\hline
\end{tabular}

Table 2. Lipinski's Rule of Five from docked ligands

\begin{tabular}{lcccc}
\hline Ligand & Molecular Weight & Log P & H-Donor & H-Acceptor \\
\hline Rosiglitazone & 357,427 & 2.15 & 1 & 5 \\
Rutin & 610,5175 & -0.87 & 10 & 16 \\
Nicotifloroside & 594,5181 & -0.57 & 9 & 15 \\
Narcissoside & 624,5441 & -0.72 & 9 & 16 \\
Quercetin & 302,2357 & 2.16 & 5 & 7 \\
Kaempferol & 286,2363 & 2.46 & 4 & 6 \\
\hline
\end{tabular}




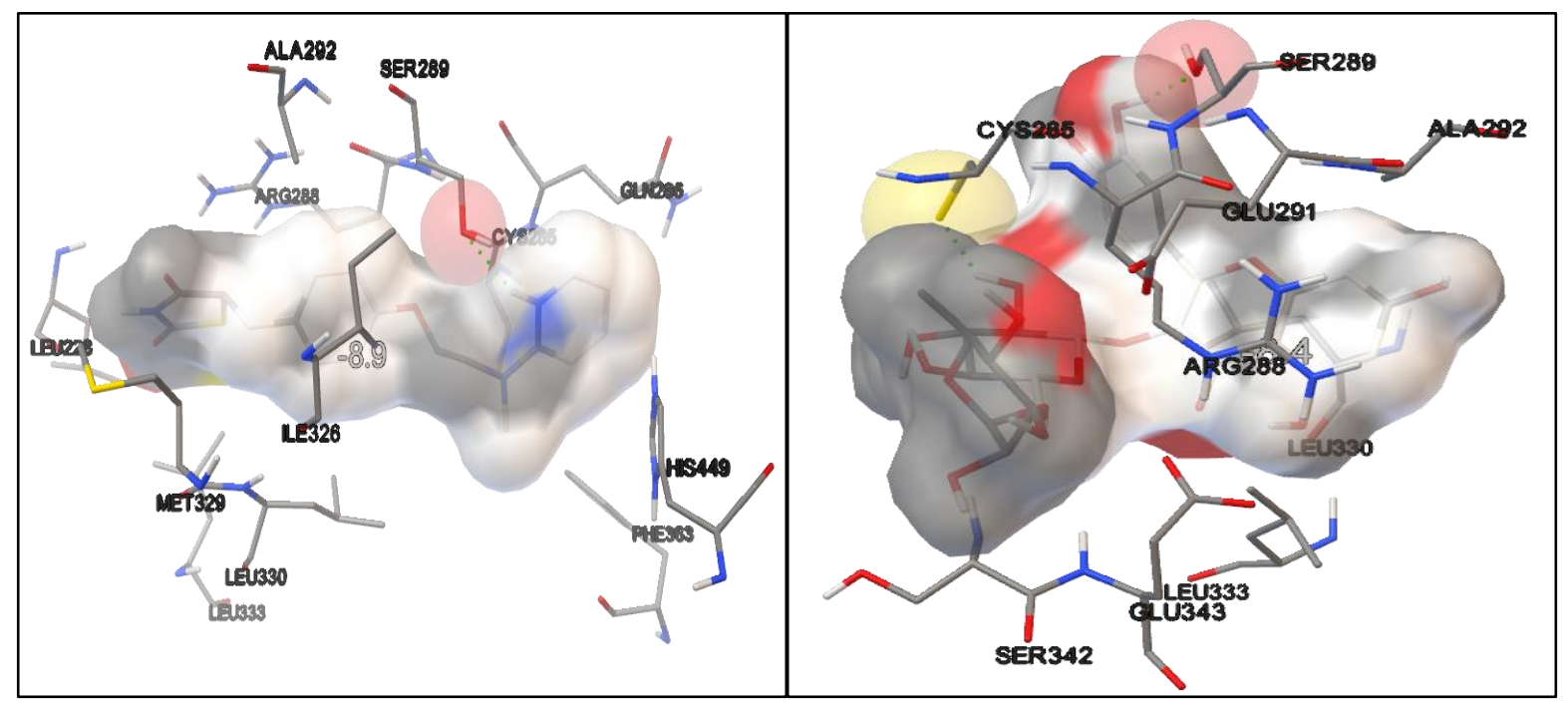

Figure 3. Visualization of Rosiglitazon interaction

Figure 4. Visualization of Rutin interaction with with residues. residues.

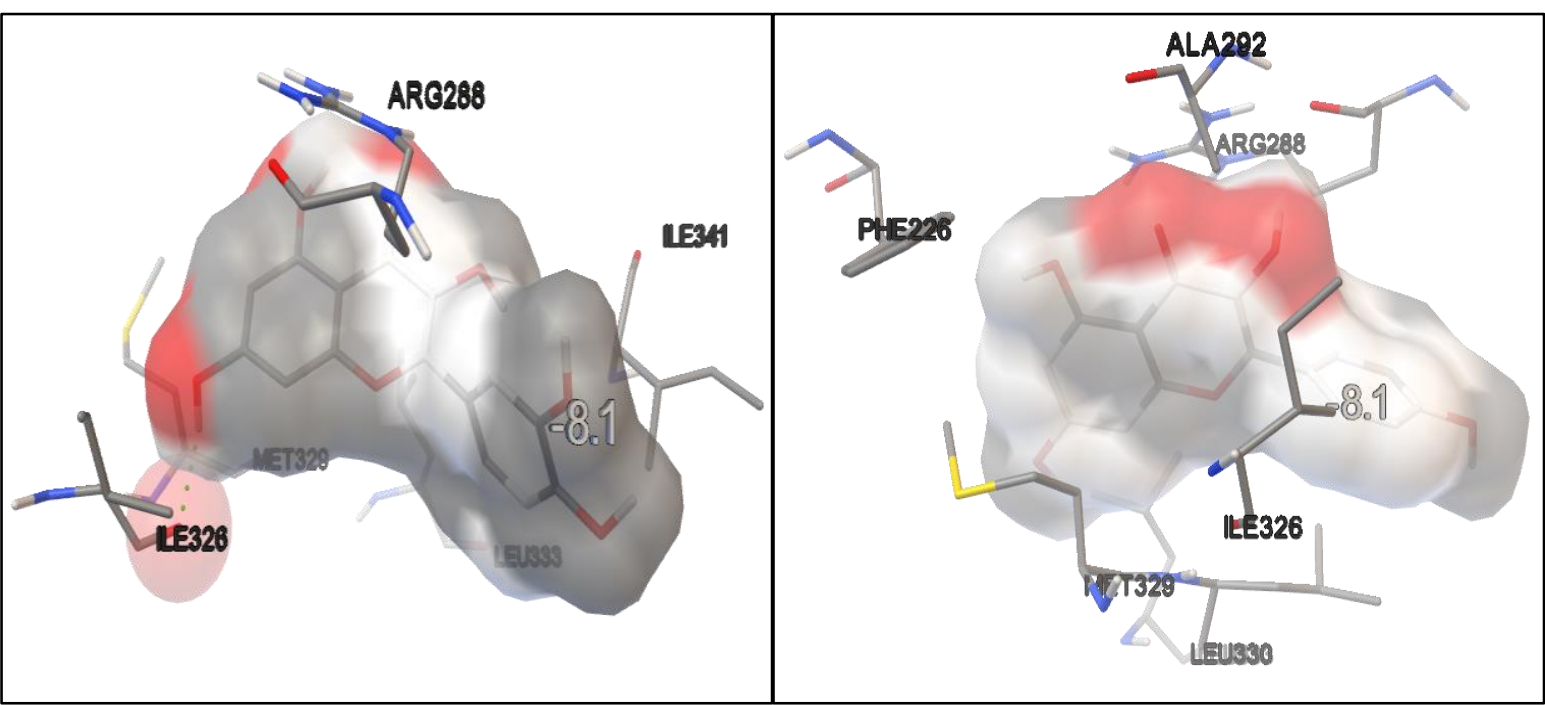

Figure 5. Visualization of Quersetin interaction with Figure 6. Visualization of Kaempferol interaction with residues. residues.

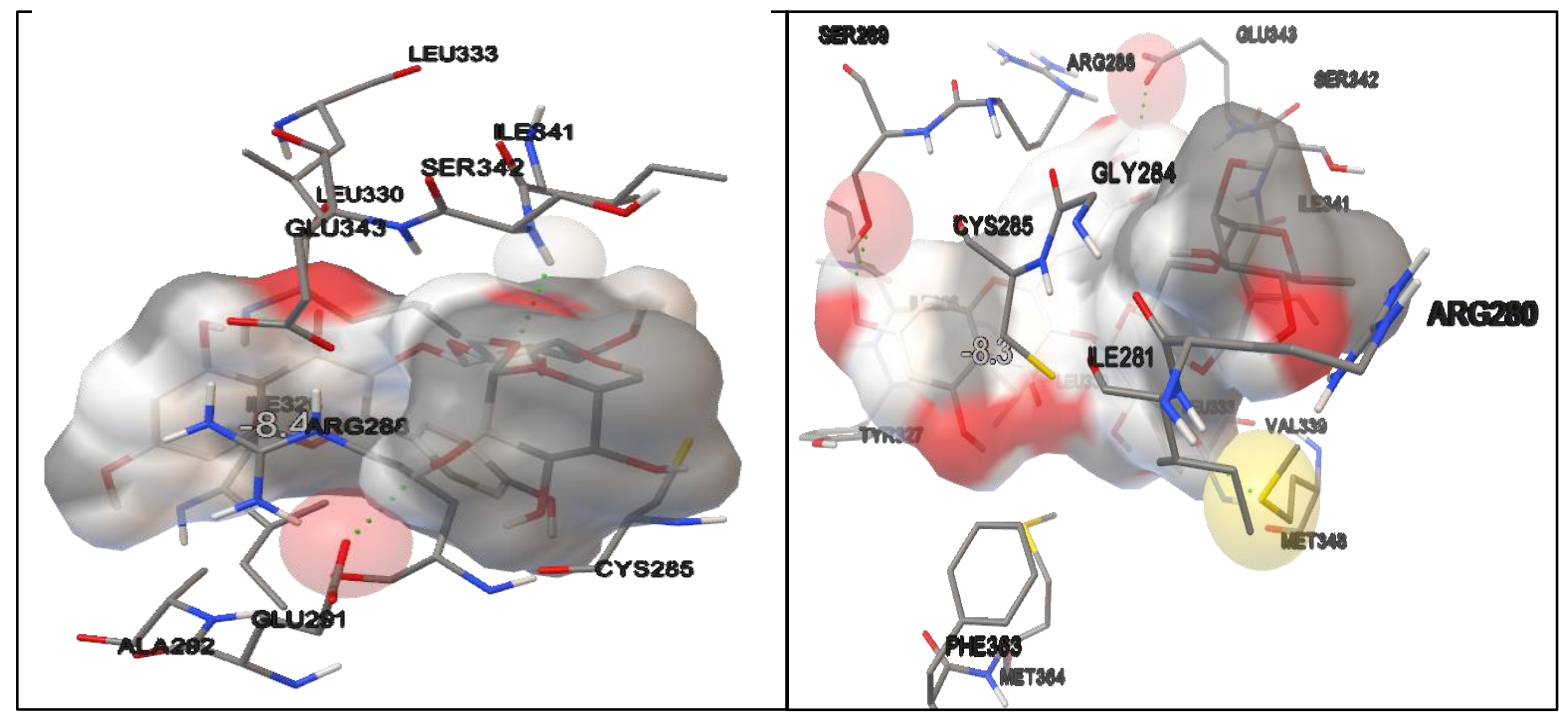

Figure 7. Visualization of nicotifloroside interaction Figure 8. Visualization of narcissosideinteraction with with residues. residues. 


\section{Conclusions}

The docking result revealed that all the selected flavonoids showed binding energy ranging between -8.1 $\mathrm{kcal} / \mathrm{mol}$ to $-8.5 \mathrm{kcal} / \mathrm{mol}$. Eventhough, when compared with rosiglitazone $(-8.9 \mathrm{kcal} / \mathrm{mol})$, the value of the binding energy of flavonoids was smaller, but close to rosiglitazone. This suggests that the flavonoid compounds have potency as antidiabetic agent.

Based on Lipinski's Rule, the five flavonoid compounds and rosiglitazone showed that from five flavonoid compounds, only quercetin and kaempferol which meets the four criteria of Lipinski's Rule, while the other three are not. This means that only quercetin and kaemperol that may be clinically active when administered orally.

\section{Acknowledgment}

We thank to Faculty of Medicine and Health Science UIN Syarif Hidayatullah for encouragement and support to carry out this work.

\section{References}

[1] N. Nordisk, Where economics and health meet: changing diabetes in Indonesia, In N. Nordisk, The Blueprint for Change Programme Indonesia, Novo Nordisk, 2013, 3.

[2] C. Coman and C. Socaciu, Docking of phytochemicals to the peroxisome proliferator-activated receptor-gamma, Bulletin UASVM Agriculture, 2012.

[3] D.R. Singh, Morinda citrifolia L. (Noni): A review of the scientific validation for its nutritional and therapeutic properties, Journal of Diabetes and Endocrinology, 3, 2012.

[4] I. Kustarini, S.S. Dewi, and I. Pawitra, Efek ekstrak etanol Morinda citrifolia L (Mengkudu), Media Medika Indonesia, 2012.

[5] J.K. DiStefano, and R.M. Watanabe, Pharmacogenetics of anti-diabetes drugs, Pharmaceuticals, 2010.

[6] W. Shen and Y.-H. Lu, Molecular docking of citrus flavonoids with some targets related to diabetes, A Journal of the Bangladesh Pharmacological Society (BDPS), (2013).

[7] G. Patrick, Instant Notes in Medicinal Chemistry, Oxford: BIOS Scientific Publisher, 2001. 